\title{
Improving the gluon condensate in three-dimensional Yang-Mills theory
}

\section{S. Caron-Huot*}

Department of Physics, McGill University, 3600 University Street, Montreal, Canada H3A 2T8 E-mail: Sicahephysics.mcgill.ca

\begin{abstract}
We apply the tadpole and cactus improvement methods to the measurement of the gluon condensate of 3D SU(3) Yang-Mills theory. The matching of the lattice measurement to the continuum $\overline{\mathrm{MS}}$ scheme involves the subtraction of a series of lattice artifacts divergent up to four loop, which has recently appeared in the literature. The two tested improvement methods are found to significantly increase the convergence to the continuum. The well-known dominance of tadpole diagrams in lattice perturbation theory is observed to persist at higher orders under the form of "cactus" diagrams.
\end{abstract}

XXIVth International Symposium on Lattice Field Theory

July 23-28, 2006

Tucson, Arizona, USA

\footnotetext{
* Speaker.
} 


\section{Introduction}

The gluon condensate of 3D Yang-Mills theory recently received interest, due to its connection with the $\mathscr{O}\left(g^{6}\right)$ non-perturbative contribution to the free energy of QCD at high temperatures (more precisely, pure glue 3D Yang-Mills is believed, through dimensional reduction, to provide an effective theory for the thermodynamics of the ultrasoft magnetic fields, $k \sim g^{2} T$, of QCD [1]). This gluon condensate was recently measured on the lattice[2], and converted to to continuum $\overline{\mathrm{MS}}$ scheme via a perturbative calculation.

A direct measurement of the gluon condensate $\left\langle 1-\frac{1}{N_{c}} \operatorname{Tr}\left[P_{12}\right]\right\rangle$ on the lattice is necessarily dominated by lattice artifacts, basically because this is a cubic divergent composite operator. However, thanks to the super-renormalizability of 3D Yang-Mills theory ( $g^{2}$ has mass dimension 1$)$, it is possible to calculate perturbatively and subtract the finite series of artifacts relevant in the $a \rightarrow 0$ limit. The required four loop matching calculation was completed in [3], providing the coefficients of the following limit:

$$
8 \frac{d_{A} N_{c}^{6}}{(4 \pi)^{4}} B_{G}=\lim _{a \rightarrow 0} \beta^{4}\left\{\left\langle 1-\frac{1}{N_{c}} \operatorname{Tr}\left[P_{12}\right]\right\rangle_{a}-\left[\frac{c_{1}}{\beta}+\frac{c_{2}}{\beta^{2}}+\frac{c_{3}}{\beta^{3}}+\frac{c_{4}}{\beta^{4}}\left(\ln \beta+c_{4}^{\prime}\right)\right]\right\}
$$

We tabulate the values of the coefficients $c_{n}$ in Table 1 . The quantity $B_{G}$ is related to the $\overline{\mathrm{MS}}$ free energy:

$$
f_{\overline{\mathrm{MS}}}=-g^{6} \frac{d_{A} N_{c}^{3}}{(4 \pi)^{4}}\left[\left(\frac{43}{12}-\frac{157}{768} \pi^{2}\right) \ln \frac{\mu_{\overline{\mathrm{MS}}}}{2 N_{c} g^{2}}+B_{G}\right]
$$

The difficulty involved in the four loop perturbative matching calculation justified the use the simplest available action, namely the standard Wilson action. The measurement of [2] were carried out at a typical value of $\beta \sim 50$, with statistical error bars of relative order $10^{-6}$. The convergence to the continuum limit was found to be rather poor; it is therefore natural to try to improve it.

\section{Tadpole improvement}

The "tadpole improvement" scheme of Lepage and Mackenzie [5] attempts to absorb lattice artifacts through a rescaling of the operators and coupling constant of the theory. It is motivated by the observation that the sum average of unitary matrices is not unitary, but is shorter. Heuristically, infrared physics may be thought of as small $\left(1+i A_{\mu}\right)$-type fluctuations living on top of link matrices which are shortened by short-distance fluctuations.

This shortening of the infrared operators can be estimated in a gauge-invariant way by measuring the expectation value of the plaquette (which is dominated by lattice-scale physics), yielding the well-known prescription:

- "Physical" plaquette: $\left(1-\frac{1}{N_{c}} \operatorname{Tr}\left[\mathrm{P}_{12}\right]\right)_{\mathrm{eff}} \equiv\left(1-\frac{1}{N_{c}} \operatorname{Tr}\left[\mathrm{P}_{12}\right]\right) /\left\langle\frac{1}{N_{c}} \operatorname{Tr}\left[\mathrm{P}_{12}\right]\right\rangle_{\mathrm{a}}$

- "Physical" coupling: $\beta_{\text {eff }} \equiv \beta\left\langle\frac{1}{N_{c}} \operatorname{Tr}\left[\mathrm{P}_{12}\right]\right\rangle_{\mathrm{a}}$

This replaces the limit (1.1) by:

$$
8 \frac{d_{A} N_{c}^{6}}{(4 \pi)^{4}} B_{G}=\lim _{a \rightarrow 0} \frac{\beta \beta_{\mathrm{eff}}^{3}}{c_{4}^{(T)}}\left\{\left\langle 1-\frac{1}{N_{c}} \operatorname{Tr}\left[P_{12}\right]\right\rangle_{a}-\left[\frac{c_{1}^{(T)}}{\beta}+\frac{c_{2}^{(T)}}{\beta \beta_{\mathrm{eff}}}+\frac{c_{3}^{(T)}}{\beta \beta_{\mathrm{eff}}^{2}}+\frac{c_{4}^{(T)}}{\beta \beta_{\mathrm{eff}}^{3}} \ln \beta_{\mathrm{eff}}+\frac{c_{4}^{\prime(T)}}{\beta \beta_{\mathrm{eff}}^{3}}\right]\right\}
$$


Since the relationship between $\beta_{\text {eff }}$ and $\beta$ is already known, from the perturbative result (1.1), it is straightforward to match the series (2.1) and (1.1). Mathematically speaking, the improved limit is just as rigorous as the unimproved one.

We find, as shown in Figure 1, that this simple prescription impressively improves the convergence to the continuum limit.

\section{Cactus resummation}

It is well known that in lattice perturbation theory, at the one loop level tadpole diagrams produce dominant contributions. In a sense this is related to the fact that they are the most ultraviolet divergent diagrams, and it is their ability to "see" the corners of the Brillouin zone which spares them from being suppressed by the ubiquitous geometrical factors of continuum perturbation theory. However, the author is not aware of a physical argument as to why the cactus diagrams (to be described below) should continue to dominate lattice perturbation theory at higher loop orders. But, at any rate, it is worth asking how well the resummation performs, because in practice it is very simple to apply.

Cactus resummation is based on the following approximation to the Schwinger-Dyson equations of motion:

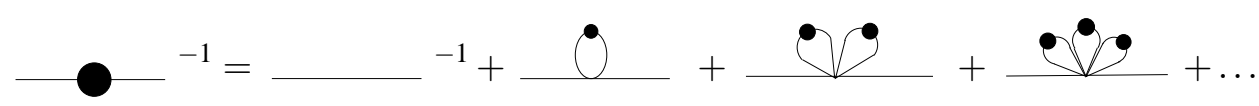

where the blob stands for the dressed propagator. An iterative solution of this equation amounts to summing an infinite series of "cactus" diagrams. The vertices on the right-hand side of (3.1) consist of only a restricted subset of the bare vertices of the theory, which can be described as follow. Using the Baker-Campbell-Hausdorff formula, the plaquette matrices can be expressed as a single exponential, which can be expanded into powers of the gauge field $A_{\mu}$ (the link matrices being $\left.U_{\mu}=\exp \left(i A_{\mu}\right)\right)$ :

$$
P_{\mu v} \Rightarrow \exp i\left(F_{\mu \nu}^{(1)}+F_{\mu \nu}^{(2)}+F_{\mu \nu}^{(3)}+\ldots\right)
$$

The Wilson action, proportional to $\left(1-\frac{1}{N_{c}} \operatorname{Tr}\left[\mathrm{P}_{\mu \nu}\right]\right) / g^{2}$, involves a sum over the traces of products of even numbers of $F^{(i)}$ 's. The vertices of (3.1) are those coming from products involving only $F_{\mu \nu}^{(1)}$,s, where $F_{\mu \nu}^{(1)}=\tilde{\partial}_{\mu} A_{\nu}-\tilde{\partial}_{\nu} A_{\mu}$ is the linearized field strength operator on the lattice.

In the cactus approximation, the dressing of the propagator is momentum independent, and the gap equation (3.1) involves only a single number, the coefficient $g_{\text {eff }}^{2}$ of the dressed propagator. Panagopoulos and Vicari [6] have shown how to perform analytically as a function of $g_{\text {eff }}^{2}$ the infinite sum on the RHS of (3.1), yielding an equation to be solved self-consistently for $g_{\text {eff }}^{2}$ (we set $a=1$ from here):

$$
\begin{aligned}
g_{o}^{2} & =\frac{-2 d g_{\text {eff }}^{2}}{N_{c}^{2}-1} \frac{d}{d g_{\text {eff }}^{2}} G\left(g_{\text {eff }}^{2}\right) \\
G\left(g_{\text {eff }}^{2}\right) & =\exp \left(-\frac{g_{\text {eff }}^{2}\left(N_{c}-1\right)}{2 d N_{c}}\right) L_{N_{c}-1}^{1}\left(\frac{g_{\text {eff }}^{2}}{d}\right)
\end{aligned}
$$

where $L_{N_{c}-1}^{1}(u)=\left(3-3 u+u^{2} / 2\right)$ for $\mathrm{SU}(3)$, is a Laguerre polynomial, and $d=3$ is the dimension of space-time. 


\subsection{An effective theory}

The cactus dressing of the inverse propagator should be applied similarly to all of the operators appearing in the action. It is found that the cactus dressing of an operator depends only on the number of $F_{\mu \nu}^{(i)}$,s out of which it is constructed. In particular, the three-point vertex and the part of the four-point vertex not involving $\operatorname{Tr}\left(F_{\mu v}^{(1)}\right)^{4}$ are all simply rescaled by the same factor $g^{2} / g_{\text {eff }}^{2}$ as the inverse propagator, because they all involve only two $F_{\mu \nu}^{(i)}$,s, suggesting to interpret $g_{\text {eff }}^{2}$ as an effective coupling constant.

However, the dressing of the unit operator and of higher-dimensional operators will be different. The dressing of the unit operator is simple to evaluate, but the effect of dressing the other operators is more difficult to account for. This suggests introducing a simple effective theory, rewriting the action via:

$$
\begin{aligned}
\frac{1-\frac{1}{N_{c}} \operatorname{Tr}\left[P_{\mu v}\right]}{g^{2}} & \left.\Rightarrow \frac{1_{\text {cactus }}}{g^{2}}+\frac{1-\frac{1}{N_{c}} \operatorname{Tr}\left[P_{\mu v}\right]}{g_{\text {eff }}^{2}}+\text { (remainder }\right) \\
1_{\text {cactus }} & =1-\frac{1}{N_{c}} G\left(g_{\text {eff }}^{2}\right)
\end{aligned}
$$

where the remainder is expanded into powers of $g_{\text {eff }}^{2} \hbar$, e.g., each extra power of $g_{\text {eff }}^{2}$ is thought of as higher order in the loop expansion. The term $1_{\text {cactus }}$ is precisely the sum of all purely "cactus" diagrams, e.g. full contractions of the vertices $\operatorname{Tr}\left(F_{\mu v}^{(1)}\right)^{2 n}$ using the dressed propagator. A loopwise calculation in this theory involves summing all diagrams up to a given number of loops, which do not contain cactus subdiagrams, except when this cactus dressing differs from the $g^{2} / g_{\text {eff }}^{2}$ rescaling ${ }^{1}$.

For the expectation value of the plaquette, we calculate the expectation value of the action (times $g^{2}$ ) in this effective theory. This yields the term $1_{\text {cactus, }}$, plus a series in powers of $g_{\text {eff }}^{2}$ multiplied by an overall $g^{2} / g_{\text {eff }}^{2}$ :

$$
\begin{aligned}
8 \frac{d_{A} N_{c}^{6}}{(4 \pi)^{4}} B_{G}=\lim _{a \rightarrow 0} \frac{\beta \beta_{\mathrm{eff}}^{3}}{c_{4}^{(c)}} & \left\{\left\langle 1-\frac{1}{N_{c}} \operatorname{Tr}\left[P_{12}\right]\right\rangle_{a}-1_{\text {cactus }}\right. \\
& \left.-\left[\frac{c_{1}^{(c)}}{\beta}+\frac{c_{2}^{(c)}}{\beta \beta_{\mathrm{eff}}}+\frac{c_{3}^{(c)}}{\beta \beta_{\mathrm{eff}}^{2}}+\frac{c_{4}^{(c)}}{\beta \beta_{\mathrm{eff}}^{3}} \ln \beta_{\mathrm{eff}}+\frac{c_{4}^{(c)}}{\beta \beta_{\mathrm{eff}}^{3}}\right]\right\}
\end{aligned}
$$

where $\beta_{\text {eff }}=2 N_{c} / g_{\text {eff }}^{2}$. Thus, because $g_{\text {eff }}^{2}$ can be expanded into an asymptotic series in $g^{2}$ with easily computable coefficients, the coefficients of the series (3.5) can be deduced from no additional input than the knowledge of the unimproved series (1.1). This nice property holds for any observable in the theory, and renders this effective theory particularly tractable from a practical viewpoint.

As mentioned above, this effective theory is not unique. For instance, one might argue that a "better" effective theory could be obtained by incorporating the extra dressing (difference from the $g^{2} / g_{\text {eff }}^{2}$ rescaling) of higher dimensional operators, at the price of making calculations more

\footnotetext{
${ }^{1}$ As is well-known, the loop expansion in this theory breaks down starting at four loop, due to infrared divergences 帏. We are ignoring this problem, focusing on lattice artifacts issues.
} 


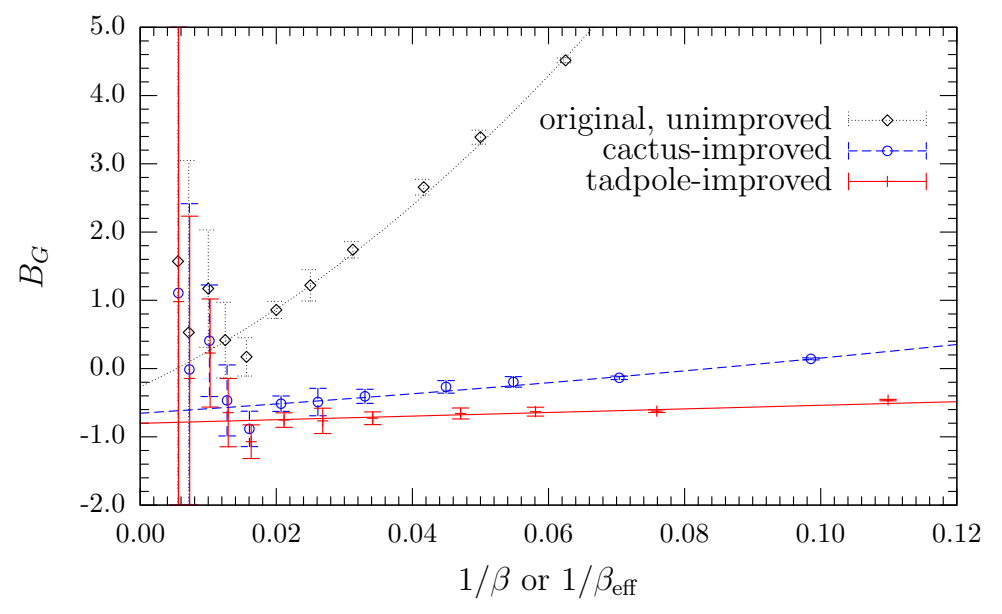

Figure 1: Comparison between the original data of [2] and its improved versions. The fits are quadratic in $\beta^{-1}$.

complicated. However, we have estimated the magnitude of the effects of such a choice, and found them to be numerically small (about an order of magnitude smaller than the error bars of Figure 1).

As exhibited by Table 1, we find that the expansion coefficients of the plaquette in the cactusimproved scheme are significantly smaller than the original ones. This means that cactus diagrams account for a numerically large fraction of the perturbative series.

\section{Results and Conclusion}

We compare the results of taking the different limits, shown on Figure 1. The plot speaks for itself: the improved limits clearly appear more convincing. The original result was $B_{G}=$ $-0.2 \pm 0.4$. Quadratic fits of the improved data yield $B_{G}=-0.6 \pm 0.3$ and $B_{G}=-0.75 \pm 2$ for the cactus- and tadpole- improved data respectively. These error estimates do not include the additional \pm 0.4 stemming from the theoretical uncertainty in the four loop coefficient $c_{4}^{\prime}$.

It is also interesting to compare the coefficients used in the various limits. They are shown in Table 1. The coefficients of the improved limits represent what is left over by the improvement scheme. The coefficients $c_{1}, c_{2}, c_{3}$ arise purely from lattice artifacts, and it is satisfying to notice that whenever they get modified by the improvement, they get significantly smaller. The coefficient of the logarithm, $c_{4}$, is universal and arises from the sensitivity of the plaquette to an infrared mass scale, provided by the scale of non-perturbative physics, $k \sim g^{2}$. No improvement scheme designed to deal with lattice artifacts is expected to be able to see it.

The coefficient $c_{4}^{\prime}$, which is the difference between the $\overline{\mathrm{MS}}$ and lattice constants next to the logarithm, receives contribution from both infrared and lattice scale physics. It is satisfying to note that it is smaller in the improvement schemes, but no particular physical significance should be attached to the fact that it is so impressively small in the cactus scheme.

The author acknowledges discussions with G. D. Moore, which motivated these investigations, and thanks K. Kajantie for having provided the numerical data which was used in the plots. 


\begin{tabular}{|c|c|c|c|}
\hline coefficient & unimproved & tadpole & cactus \\
\hline$c_{1}$ & $8 / 3$ & $8 / 3$ & 0 \\
\hline$c_{2}$ & $1.951315(2)$ & $1.951315(2)$ & $-0.270907(2)$ \\
\hline$c_{3}$ & $6.8612(2)$ & $1.6577(2)$ & $1.7818(2)$ \\
\hline$\left(c_{4}\right)$ & $2.92942132 \ldots$ & $2.92942132 \ldots$ & $2.92942132 \ldots$ \\
\hline$c_{4}^{\prime}$ & $7.0(3)$ & $-1.7(3)$ & $-0.3(3)$ \\
\hline
\end{tabular}

Table 1: The coefficients used in the unimproved and improved limits.

\section{References}

[1] K. Kajantie, M. Laine, K. Rummukainen and Y. Schroder, Phys. Rev. D 67, 105008 (2003) [hep-ph/0211321].

[2] A. Hietanen, K. Kajantie, M. Laine, K. Rummukainen, and Y. Schröder, JHEP 01 (2005) 013 [hep-lat/0412008]

[3] F. Di Renzo, M. Laine, V. Miccio, Y. Schröder and C. Torrero, JHEP 0607, 026 (2006) [hep-ph/0605042]

[4] A. D. Linde, Phys. Lett. B 96, 289 (1980).

[5] G.P. Lepage and P.B. Mackenzie, Phys. Rev. D 48 (1993) 5 [hep-lat/920 9022 ]

[6] H. Panagopoulos and E. Vicari, Phys. Rev. D58:114501 (1998) [hep-lat/9806009] 